\title{
Evolutionary Game Theory: A Generalization of the ESS Definition
}

\author{
Elvio Accinelli \\ Universidad Autónoma de San Luis Potosí \\ Álvaro Obregón \#64, Col. Centro, C. P. 78000 \\ San Luis Potosí, S. L. P., Mexico \\ elvio.accinelli@eco.uaslp.mx \\ Filipe Martins \\ Department of Mathematics, Faculdade de Ciências da \\ Universidade do Porto, Rua do Campo \\ Alegre, s/n, 4169-007 Porto, Portugal \\ Laboratório de Inteligência Artificial e \\ Apoio à Decisão (LIAAD), INESC TEC \\ Campus da FEUP, Rua Dr. Roberto Frias \\ 4200-465 Porto, Portugal \\ philip_m90@hotmail.com \\ Jorge Oviedo* \\ Instituto de Matemática Aplicada San Luis \\ Universidad Nacional de San Luis \\ Ejército de los Andes 950, 5700 San Luis, Argentina \\ Consejo Nacional de Investigaciones \\ Científicas y Técnicas (CONICET) \\ Ejército de Los Andes 950 5700, San Luis, Argentina \\ joviedo@unsl.edu.ar \\ Received 3 November 2017 \\ Revised 23 March 2018 \\ Accepted 6 April 2019 \\ Published 28 June 2019
}

In this paper, we study the concept of Evolutionarily Stable Strategies (ESSs) for symmetric games with $n \geq 3$ players. The main properties of these games and strategies are analyzed and several examples are provided. We relate the concept of ESS with previous literature and provide a proof of finiteness of ESS in the context of symmetric games with $n \geq 3$ players. We show that unlike the case of $n=2$, when there are more than two populations an ESS does not have a uniform invasion barrier, or equivalently, it is not equivalent to the strategy performing better against all strategies in a neighborhood.

* Corresponding author. 
We also construct the extended replicator dynamics for these games and we study an application to a model of strategic planning of investment.

Keywords: Evolutionary stable strategy; $n$-symmetric games; invasion barriers; local superior strategies; dynamic stability.

\section{Introduction}

The concept of Evolutionarily Stable Strategy (denoted ESS) was defined in Mavnard Smith and Price 1973 for symmetric bimatrix games. The work of Maynard Smith 1982 is an excellent reference to the theory of games and evolution. Bromm et al. [1997], Bukowski and Miekisz [2004] and Palm [1984] extended the concept of Evolutionarily Stable Strategy for symmetric $n$-player games. When animals compete for limited resources, an ESS gives a response such that no mutant population can invade the population that adopts the ESS. The concept of ESS was also extended to asymmetric games (see Weibull [1995]). Gokhale and Traulsen 2010] present a study of games with more than two players highlighting the difficulties of their mathematical modeling.

Evolutionary game theory has been used to model the evolution of biologically relevant scenarios such as microbial diversity but also in other areas such as economics as well as the evolution of social dilemmas. The relevance of games with multiple populations and multiple strategies as a tool to understand social behavior has been highlighted by several biologists, particularly in studies of evolution, but also economists and sociologists interested in dynamical evolutionary models and structural change. Most of the theories of evolutionary games have been developed on the basis of a population facing itself or of two or more, populations with antagonistic interests in the case of asymmetric games, see, for instance Accinelli and Carrera 2011] and also Accinelli et al. 2015] where the authors introduce a model to study poverty traps and the relations between firms and works that invest in technology and education. Many conflicts in nature or in society confront diverse populations or individuals, each one with multiple possible strategies they can choose to solve them. Cooperation or competition chosen as a strategy to resolve a conflict that faces multiple populations determines its future developments. For instance, economic models that refer to conflicts due to the existence of externalities or public goods require the partaking of many different groups of individuals with conflicting interests, who may or may not collaborate to solve the conflict. See the work of Kurokawa and Ihara 2009] where they provide a model to study the emergence of cooperation in public good games. In biology, the importance of the analysis of conflicts involving multiple populations with multiple strategies to the study of evolution is widely recognized, for instance, in van Veelen 2009].

In this paper, we study an extension of the classic definition of ESS to symmetric games with $n \geq 3$ populations following the definitions in Palm 1984] and Bromm et al. [1997]. In other words, we have that a population is playing against several copies of itself, or in other way that $n \geq 3$ players are drawn at random 
from a population and play a game. This definition differs from the classic multipopulation definition of ESS in the sense that for the latter the mutations that try to invade the population have different sizes. In Definition 5.1 in Chap. 5 of Weibull [1995], there is a very general definition of ESS for multi-population games which is equivalent to the concept of Strict Nash Equilibrium. Not all multi-population games have Strict Nash Equilibrium and on the other hand this claim says that the concept of ESS does not add anything new to the theory. The definition of ESS we study is more general than the concept of Strict Nash Equilibrium. In this definition, in accordance to symmetric games, the mutations that try to invade the population also reflect the symmetry. We show some examples of ESS that exhibit that this concept is weaker than the Strict Nash Equilibrium and an example that disproves one of the original characterizations of ESS presented in Palm 1984. We also show an example of an ESS where there is no uniform invasion barrier, i.e., a uniform barrier on the mutation sizes in the sense that it does not depend on the mutation itself, unlike the classic $n=2$ case where the concepts of ESS and ESS with uniform invasion barrier are equivalent. Nevertheless, we show that when there are only two pure strategies, regardless of the number of players, these two concepts are equivalent. We also show that the set of ESS is finite or possibly an empty set. Furthermore, we consider the replicator dynamics and we provide an example of an ESS that is not asymptotically stable.

Recall that it is possible to interpret each player as a population, and the mixed strategy followed by a player as a distribution of the population over a set of possible behaviors. As we have indicated this population approach is appropriate to explain different economical conflicting situations. As specific examples, we refer to the oligopolistic competitive behaviors, or the choice of a portfolio in finance theory. As it is well known, the term oligopoly refers to a market or an industry branch dominated by a small number of sellers or producers (oligopolists). Oligopolies can result from various forms of collusion which reduce competition and lead to higher costs for consumers. With our approach it is possible to model the behavior of an oligopolistic market where each firm can produce the same different types of product, each one destined to different segments of the oligopolized market. For example, in the market of cars, different companies produce cars of different ranges, destined to different segments of the market. However, basically the characteristics within each range are the same, regardless of the firm that produces. In Sec. 4.2 we will consider an example from finance theory. As it is usual to consider in this theory, we introduce a population of investors with similar characteristics looking to maximize the performance of their investments in assets. In this example a population of investors competes against itself. The set of assets is considered the set of pure strategies, and the set of possible portfolios as the set of mixed strategies. The choice of each investor will have an impact on the values of these assets and consequently on the returns of each portfolio. Strategic planning for each investor needs to take into account the likely responses of the competitors. 
The rest of the paper is organized as follows. In Sec. 2 we introduce the basic definitions of the $n$-player symmetric games. Next, in Sec. 3 we analyze the structure of the ESS set, and we study the main properties of the definition. We prove that the ESS set is finite and provide some examples illustrating how results change from the $n=2$ case and for the general nonsymmetrical version of the definition. In Sec. 4 we introduce a generalized replicator dynamics for a multi-population multi-strategic game that is played continuously in time. Finally, we introduce a finance problem where several identical investors are choosing the best portfolio given that the others are doing it. We conclude in Sec. 5.

\section{Basic Definitions}

We restrict our attention to symmetric and finite normal form games. By $N=$ $\{1, \ldots, n\}$ we denote the set of players. For each player, indexed by $i \in N$, let $S_{i}$ be her finite set of pure strategies. We denote by $\mathbf{S}=S_{1} \times \cdots \times S_{n}$ the set of pure strategic profiles. The payoff function is $\pi=\left(\pi_{1}, \ldots, \pi_{n}\right)$ where $\pi_{i}: \mathbf{S} \rightarrow \mathbb{R}$. The game generated by $\mathbf{S}$ and $\pi$ is denoted by $(N, \mathbf{S}, \boldsymbol{\pi})$. The space of mixed strategies is $\Delta \mathbf{S}=\prod_{i=1, \ldots, n} \triangle\left(S_{i}\right)$ where $\Delta\left(S_{i}\right), i=1, \ldots, n$, by denotes the set of probabilities over $S_{i}$. We extend the payoff function $\pi$ to $\Delta \mathbf{S}$ linearly as usual. We will often fail to differentiate between the pure strategy $s_{j}^{i}$ of player $i$ and the mixed strategy for $i$ which places full probability on $s_{j}^{i}$. We will say that a strategy is symmetric if every player chooses the same strategy (pure or mixed).

Given a pure strategy profile $\mathbf{s}=\left(s_{1}, \ldots, s_{n}\right) \in \mathbf{S}$, a mixed strategy profile $\boldsymbol{\sigma}=\left(\sigma_{1}, \ldots, \sigma_{n}\right)$ gives rise to $\boldsymbol{\sigma}(\mathbf{s})=\left(\sigma_{1}\left(s_{1}\right), \ldots, \sigma_{n}\left(s_{n}\right)\right)$, where $\sigma_{i}\left(s_{i}\right)$ denotes the probability that player $i$ plays its pure strategy $s_{i} \in S_{i}$, when he is playing according to mixed strategy $\sigma_{i} \in \Delta\left(S_{i}\right)$. The carrier or support of the mixed strategy profile $\boldsymbol{\mu}=\left(\mu_{1}, \ldots, \mu_{n}\right) \in \Delta \mathbf{S}$ is $C(\boldsymbol{\mu})=C\left(\mu_{1}, \ldots, \mu_{n}\right)=\left(C_{1}\left(\mu_{1}\right), \ldots, C_{n}\left(\mu_{n}\right)\right)$, where

$$
C_{i}\left(\mu_{i}\right)=\left\{s_{i} \in S_{i}: \mu_{i}\left(s_{i}\right)>0\right\} .
$$

$\boldsymbol{\mu}=\left(\mu_{1}, \ldots, \mu_{n}\right)$ is said to be completely mixed strategy if for all $i \in N, C_{i}\left(\mu_{i}\right)=S_{i}$.

Let $\boldsymbol{\sigma}=\left(\sigma_{1}, \ldots, \sigma_{n}\right)$ and $\boldsymbol{\mu}=\left(\mu_{1}, \ldots, \mu_{n}\right)$ be two strategy profiles and let $t, k$ be two natural numbers. We will use the following notation:

$$
\left(\boldsymbol{\sigma}_{-[t, k]}, \boldsymbol{\mu}\right)= \begin{cases}\left(\sigma_{1}, \ldots, \sigma_{t-1}, \mu_{t}, \ldots, \mu_{k}, \sigma_{k+1}, \ldots, \sigma_{n}\right) & \text { if } t \leq k, \\ \left(\sigma_{1}, \ldots, \sigma_{n}\right) & \text { if } t>k .\end{cases}
$$

We use the simplified notation $\left(\boldsymbol{\sigma}_{-i}, \boldsymbol{\mu}\right)=\left(\boldsymbol{\sigma}_{-[i, i]}, \boldsymbol{\mu}\right)$, for $i=1, \ldots, n$. As usual, if $\boldsymbol{\sigma}$ is the strategy profile $\boldsymbol{\sigma}=\left(\sigma_{1}, \ldots, \sigma_{n}\right)$ we denote by $\boldsymbol{\sigma}_{-i}$ the profile $\boldsymbol{\sigma}_{-i}=$ $\left(\sigma_{1}, \ldots, \sigma_{i-1}, \sigma_{i+1}, \ldots, \sigma_{n}\right)$.

The best response correspondence of player $i \in N$ is given by

$$
B_{i}\left(\boldsymbol{\sigma}_{-i}\right)=\left\{\mu \in \Delta\left(S_{i}\right): \pi_{i}\left(\sigma_{-i}, \mu\right) \geq \pi_{i}\left(\sigma_{-i}, \mu^{\prime}\right) \quad \text { for all } \mu^{\prime} \in \Delta\left(S_{i}\right)\right\},
$$

and the best response correspondence $B: \Delta \mathbf{S} \rightarrow \Delta \mathbf{S}$ is given by

$$
B(\boldsymbol{\mu})=B\left(\mu_{1}, \ldots, \mu_{n}\right)=B_{1}\left(\boldsymbol{\mu}_{-1}\right) \times \cdots \times B_{n}\left(\mu_{-n}\right) .
$$


Let $(N, \mathbf{S}, \pi)$ be a game. A strategy profile $\boldsymbol{\sigma}=\left(\sigma_{1}, \ldots, \sigma_{n}\right)$ is a Nash Equilibrium if and only if for all $i \in N$ we have $\sigma_{i} \in B_{i}\left(\sigma_{-i}\right)$.

Definition 1. $(N, \mathbf{S}, \pi)$ is an $n$-player symmetric game if every player has the same strategy space and for every permutation of the set of players, $\theta: N \rightarrow N$, we have that $\pi_{i}\left(s_{1}, \ldots, s_{n}\right)=\pi_{\theta(i)}\left(s_{\theta(1)}, \ldots, s_{\theta(n)}\right)$.

For symmetric games it is enough to specify the payoff function of a given player, such as, say, player 1 . We will denote by $\Gamma=(N, S, \pi)$ the $n$-symmetric game $(N, \mathbf{S}, \pi)$ where $\mathbf{S}=S \times \cdots \times S$ and $\pi=\left(\pi_{1}, \ldots, \pi_{n}\right)$, satisfying the definition of symmetry and $\pi=\pi_{1}$.

Remark 1. If $N=2$ this definition coincides with the standard definition of two-player symmetric games. If $N=3$ we have that $\pi_{1}\left(s_{1}, s_{2}, s_{3}\right)=\pi_{1}\left(s_{1}, s_{3}, s_{2}\right)$, because $\pi_{1}\left(s_{1}, s_{2}, s_{3}\right)=\pi_{2}\left(s_{2}, s_{1}, s_{3}\right)=\pi_{3}\left(s_{2}, s_{3}, s_{1}\right)=\pi_{1}\left(s_{1}, s_{3}, s_{2}\right)$. Other similar relations may be obtained. In general, we have that the payoff of a player remains the same if the two remaining players change their strategies.

Remark 2. Nash 1951] showed that every symmetric game has a symmetric Nash Equilibrium, i.e., a Nash Equilibrium where every player chooses the same strategy. For this he considered the best response correspondence $\beta: \Delta S \rightarrow \Delta S$ given by

$$
\beta(\boldsymbol{\sigma})=\left\{\mu \in \Delta S: \pi\left(\mu, \boldsymbol{\sigma}_{-i}\right) \geq \pi\left(\mu^{\prime}, \boldsymbol{\sigma}_{-i}\right) \text { for all } \mu^{\prime} \in \Delta S\right\},
$$

where $\boldsymbol{\sigma}=(\sigma, \ldots, \sigma)$, and showed that it has a fixed point. This fixed point corresponds to a Symmetric Nash Equilibrium, i.e., a Nash Equilibrium where all players play the same strategy.

From here on we will work with symmetric games. Suppose that a small group of mutants appears in a large population of individuals, all of whom are playing according to the same (mixed or pure) strategy $\sigma \in \Delta S$. Suppose that a mutation represented by a strategy $\mu \neq \sigma \in \Delta S$ tries to invade the population. Let $\epsilon \in(0,1)$ be the share of mutants in the post-entry population, i.e., the population after the mutant invasion. Suppose that groups of $n$ individuals are repeatedly drawn at random to play the game, each individual being drawn with equal probability. So, if an individual is drawn to play the game, then the probability that each opponent plays the mutant strategy is $\epsilon$ and the probability that he plays the incumbent strategy is $(1-\epsilon)$. The payoff in this $n$-player game is the same as in this each opponent plays the mixed strategy $\mu(\epsilon)=(1-\epsilon) \sigma+\epsilon \mu \in \Delta S$. The post-entry payoff of the incumbent strategy is $\pi(\sigma, \mu(\epsilon), \ldots, \mu(\epsilon))$ and that of the mutant strategy is $\pi(\mu, \mu(\epsilon), \ldots, \mu(\epsilon))$.

Following Palm 1984] and Bromm et al. [1997] we consider the next definition.

Definition 2. Let $\Gamma$ be a symmetric game, a symmetric strategy, $\boldsymbol{\sigma}=(\sigma, \ldots, \sigma)$, is an ESS (or simply $\sigma \in \Delta S$ is an ESS) if for all $\mu \in \Delta S$ such that $\mu \neq \sigma$ there 
exists $\epsilon_{\mu} \in(0,1)$ such that for all $\epsilon \in\left(0, \epsilon_{\mu}\right)$ we have

$$
\pi(\sigma, \mu(\epsilon), \ldots, \mu(\epsilon))>\pi(\mu, \mu(\epsilon), \ldots, \mu(\epsilon)),
$$

where $\mu(\epsilon)=(1-\epsilon) \sigma+\epsilon \mu$.

The definition of ESS above differs from the usual definition for multi-population games (see, for instance, Definition 5.1 in Chap. 5 of Weibull [1995]) or that suggested in Taylor [1979]), because in our framework only the same kind of mutations in each population makes sense, and moreover this mutation occurs with the same size $\epsilon$ in each population. In the framework of symmetric games this is a natural definition, since given that all populations are the same they have the same probabilities to be invaded by the same kind of mutation. More precisely, in Definition 5.1 in Weibull [1995], the post-entry strategy is allowed to be $\mu_{i}(\epsilon)=(1-\epsilon) \sigma+\epsilon \mu_{i}$, with the mutation strategy $\mu_{i}$ being possibly different for each population.

An equivalent definition is given in the next proposition whose proof can be seen in Bromm et al. 1997.

Proposition 1. Let $\Gamma$ be a symmetric game, a symmetric strategy, $\sigma$, is an ESS if and only if for all $\mu \in \Delta S$ such that $\mu \neq \sigma$ :

(1) $\boldsymbol{\sigma}=(\sigma, \ldots, \sigma)$ is a Symmetric Nash Equilibrium, i.e., $\pi(\boldsymbol{\sigma}) \geq \pi\left(\left(\boldsymbol{\sigma}{ }_{-1}, \boldsymbol{\mu}\right)\right)$, where $\boldsymbol{\mu}=(\mu, \ldots, \mu)$, or equivalently $\pi(\sigma, \ldots, \sigma) \geq \pi(\mu, \sigma, \ldots, \sigma)$.

(2) There is a $k$ with $1 \leq k \leq n-1$ such that for all $j$ with $1 \leq j \leq k$,

$$
\pi\left(\left(\boldsymbol{\sigma}_{-[2, j]}, \boldsymbol{\mu}\right)\right)=\pi\left(\left(\boldsymbol{\sigma}_{-[1, j]}, \boldsymbol{\mu}\right)\right)
$$

and $\pi\left(\left(\boldsymbol{\sigma}_{-[2, k+1]}, \boldsymbol{\mu}\right)\right)>\pi\left(\left(\boldsymbol{\sigma}_{-[1, k+1]}, \mu\right)\right)$.

This proposition says (as in the case of a symmetric bimatrix game) that if $\sigma$ is an $E S S$ and if another strategy $\boldsymbol{\mu}=(\mu, \ldots, \mu)$ is an alternative best response to $\sigma$, then $\sigma$ has to be a better reply to $\mu$ than $\mu$ to itself or one of the combinations in condition (2) where $j-1$ opponent players play $\mu$. Using the nomenclature in Bromm et al. [1997] we say that an ESS satisfying condition (1) strictly is an ESS of level zero. In other words, ESS of level zero is the Strict Nash Equilibrium of the game. Otherwise, an ESS satisfying condition (2) is called an ESS of level $k$. This proposition inductively extends the definition of ESS for two populations. Indeed, this version of the definition for symmetric two-population games is the original one proposed in Maynard Smith and Price [1973].

\section{The Structure and Properties of the Set of ESSs}

In this section we will study some features of the definition of ESS above. We start with effectively showing that the definition is more general in the sense that this concept is not equivalent to Strict Nash Equilibrium. We then show that the ESS set is finite. 


\subsection{ESS and Nash Equilibria}

For Definition 5.1 in Weibull [1995] the two concepts of ESS and Strict Nash Equilibrium are shown to be equivalent. In general, as apparent from Proposition 1 , although a Strict Nash Equilibrium is always an ESS, these concepts are not necessarily equivalent. The following example also shows this fact by exhibiting a completely mixed ESS, which is obviously not a Strict Nash, since a Strict Nash is always pure.

Example 1. Consider the following symmetric 3-player game:

$$
\left[\begin{array}{cr}
-2 & 0 \\
0 & -1
\end{array}\right] \quad\left[\begin{array}{rr}
0 & -1 \\
-1 & -1
\end{array}\right] \text {. }
$$

The strategy profile is $(\sigma, \sigma, \sigma)$ where $\sigma=\left(\frac{1}{2}, \frac{1}{2}\right)$ is a Mixed Nash Equilibrium. We have that for all $\mu=\left(\mu_{1}, 1-\mu_{1}\right) \in \Delta S, \pi(\mu, \sigma, \sigma)=-3 / 4$, and $\pi(\mu, \mu, \sigma)=$ $-\mu_{1}^{2}+\mu_{1}-1$ and $\pi(\sigma, \mu, \sigma)=-3 / 4$. Since $-\mu_{1}^{2}+\mu_{1}-1<-3 / 4$ for all $\mu \neq \sigma$, we have that $\sigma$ is an ESS by Proposition 1 .

van Damme [1987] showed that for symmetric bimatrix games if $\sigma$ is an ESS then there is no other Symmetric Nash Equilibrium $\mu$ such that $C(\mu) \subseteq \beta(\sigma)$. This previous property can be generalized in the following lemma.

Lemma 1. If $(\sigma, \sigma, \sigma)$ and $(\mu, \mu, \mu)$ are Symmetric Nash Equilibria $(\sigma \neq \mu)$, $(\mu, \mu, \sigma)$ is a Nash Equilibrium and $C(\mu) \subset \beta(\sigma)$, then $\sigma$ is not an ESS.

Proof. Assume that $\boldsymbol{\sigma}=(\sigma, \sigma, \sigma)$ is an ESS. Then $\boldsymbol{\sigma}$ is a Nash Equilibrium so that $\pi(\sigma, \sigma, \sigma) \geq \pi(\mu, \sigma, \sigma)$. Since $C(\mu) \subset \beta(\sigma)$ then $\pi(\mu, \sigma, \sigma) \geq \pi(\sigma, \sigma, \sigma)$. So $\pi(\sigma, \sigma, \sigma)=\pi(\mu, \sigma, \sigma)$. Since $\boldsymbol{\sigma}$ is an ESS, from Proposition 1 we have that $\pi(\sigma, \mu, \sigma) \geq \pi(\mu, \mu, \sigma)$, and by hypothesis $(\mu, \mu, \sigma)$ is a Nash Equilibrium so $\pi(\mu, \mu, \sigma) \geq \pi(\sigma, \mu, \sigma)$. So we get $\pi(\sigma, \mu, \sigma)=\pi(\mu, \mu, \sigma)$. Since $\sigma$ is an ESS from Proposition 1 we get $\pi(\sigma, \mu, \mu)>\pi(\mu, \mu, \mu)$ and this contradicts that $(\mu, \mu, \mu)$ is a Nash Equilibrium. So $\sigma$ is not an ESS.

Equivalently, the lemma says that if $\sigma$ is an $\operatorname{ESS}$ and $(\mu, \sigma, \sigma)$ is a Nash Equilibrium with $\mu \neq \sigma$ and $C(\mu) \subset \beta(\sigma)$, then $\mu$ cannot be a Symmetric Nash Equilibrium. The previous lemma can be generalized to games with more than two players but the notation and hypothesis will become cumbersome. The following example shows that when $(\mu, \mu, \sigma)$ is not a Nash Equilibrium in the previous Proposition then $\sigma$ can be an ESS. Furthermore, we have an example of a nonstrict pure ESS.

Example 2. Consider the following symmetric 3-player game, where player one chooses rows, player two chooses columns and player three chooses matrices:

$$
\left[\begin{array}{ll}
1 & 1 \\
1 & 0
\end{array}\right] \quad\left[\begin{array}{ll}
1 & 0 \\
0 & 2
\end{array}\right] .
$$


Consider $\sigma=(1,0)$ and $\mu=(1 / 2,1 / 2)$. Note that $\boldsymbol{\sigma}=(\sigma, \sigma, \sigma)$ is a Symmetric Nash Equilibrium because $\pi(\nu, \sigma, \sigma)=1$, for all $\nu \in \Delta S$. Furthermore, it satisfies $C(\mu) \subseteq \beta(\sigma)$. Additionally, $\boldsymbol{\mu}=(\mu, \mu, \mu)$ is a Symmetric Nash Equilibrium because $\pi(\nu, \mu, \mu)=3 / 4$ for all $\nu \in \Delta S$. Since $\pi(\sigma, \mu, \sigma)=1$ and $\pi(\mu, \mu, \sigma)=3 / 4<1$ we have that $(\mu, \mu, \sigma)$ is not a Nash Equilibrium. To see that $\sigma$ is an ESS, notice that $\pi(\nu, \sigma, \sigma)=1$, for all $\nu=\left(\nu_{1}, 1-\nu_{1}\right) \in \Delta S$, and $\pi(\nu, \nu, \sigma)=\nu_{1}\left(2-\nu_{1}\right) \leq$ $\pi(\sigma, \nu, \sigma)=1$, with equality if and only if $\nu_{1}=1$, i.e., $\nu=\sigma$. By Proposition 1 , $\sigma$ is an ESS.

In the following example, we show that one of the assertions in Palm 1984], namely the equivalence between (i) and (ii) in Proposition 4 in the above reference, is wrong. Indeed, it asserts that for instance when $n=3$ for $\sigma$ to be an ESS then it must be an ESS of either level zero or one. Indeed, Proposition 1 provides the correction of this assertion in Palm [1984] since it is possible to have an ESS of level two. The reason for this fact is that when $n=3$ the dependence on $\epsilon$ in Eq. (11) is quadratic and not linear as in the $n=2$ case, and so the corresponding assertion in Palm [1984] is only valid when $n=2$.

Example 3. Consider the following symmetric 3-player game:

$$
\left[\begin{array}{ll}
1 & 1 \\
1 & 1
\end{array}\right] \quad\left[\begin{array}{ll}
1 & 1 \\
1 & 0
\end{array}\right] .
$$

The strategy profile $\sigma=(1,0)$ is a Nash Equilibrium since $\pi(\nu, \sigma, \sigma)=1$ for all $\nu \in \Delta S$. Furthermore, one also has $\pi(\sigma, \nu, \sigma)=\pi(\nu, \nu, \sigma)$ for all $\nu \in \Delta S$. Since $\pi(\sigma, \mu, \mu)-\pi(\mu, \mu, \mu)=\left(1-\nu_{1}\right)^{3}>0$ for all $\nu \neq \sigma$, we conclude by Proposition 1 that $\sigma$ is an ESS.

\subsection{Invasion barriers and local superior strategies}

Let us now introduce the definitions of locally superior strategy (LSS) and ESS with uniform invasion barrier for a symmetric multi-population game.

Definition 3. A strategy $\sigma \in \Delta S$ is an LSS if there exists a neighborhood $U$ of $\sigma$ such that for every $x \in U$, with $x \neq \sigma$,

$$
\pi(\sigma, x, \ldots, x)>\pi(x, x, \ldots, x) .
$$

Definition 4. We say that $\sigma$ is an ESS with uniform invasion barrier if in Definition 2 the inequality defining an ESS holds for all $\epsilon \in(0, \bar{\epsilon})$ where $\bar{\epsilon}>0$. In other words, the maximum size of the invading mutation into the population does not depend on the mutant invading strategy.

In Palm 1984 it is shown that these two concepts are equivalent, which also appears in Bukowski and Miekisz [2004]. The proof of this statement can be obtained using the same argument as the proof of the $n=2$ case shown in Proposition 2.6 in Weibull [1995]. 
The concept of uniform invasion barrier has not always been emphasized in previous definitions of ESS in the literature, and in some cases it has not been made clear in the definition. The definition of ESS with uniform invasion barrier is basically a reversal of the order of quantifiers related to the mutation and the maximum mutation size in Definition 2, Indeed, a careful reading of the definitions of ESS in Palm [1984] and Bromm et al. [1997] shows that a uniform invasion barrier is part of the definition of ESS. However, it is worth noting that the proof of Proposition 1 does not require the hypothesis of uniform invasion barrier and so an alternative characterization of ESS is provided in Definition 2.

Furthermore, it is known that in classic $n=2$-player symmetric games an ESS necessarily has a uniform invasion barrier and so these three concepts, ESS, ESS with uniform invasion barrier and LSS, are equivalent. The next proposition shows it is also true for two-strategy symmetric game regardless of the number of players.

Proposition 2. In two-strategy symmetric games, the concepts of ESS, ESS with uniform invasion barrier and LSS are equivalent.

Proof. It remains to prove that an ESS is also an LSS. In two-player games the payoffs of a player, say player 1, depend only on the strategy of the player and on the number of opponent players who choose a given pure strategy. Using the notation in Bromm et al. [1997] we denote by $a_{i, j}$ the payoff to player 1 when he/she chooses strategy $i \in\{1,2\}$ and exactly $j \in\{0, \ldots, n-1\}$ of the opponent $n-1$ players choose strategy 1 . Define $\beta_{j}=a_{1, j}-a_{2, j}$.

Consider the pure equilibria $\sigma=(1,0)$. After some algebraic manipulations we get that if $x=\left(x_{1}, 1-x_{1}\right)=(1-\delta, \delta)$, for $\delta \gtrsim 0$, i.e., $x \approx \sigma$, then

$$
\Pi(x)=\pi(\sigma, x, \ldots, x)-\pi(x, x, \ldots, x)=\delta\left(\sum_{i=0}^{n-1} C_{i}^{n-1}(1-\delta)^{n-1-i} \delta^{i} \beta_{i}\right) .
$$

In Bromm et al. [1997] it is proven that $\sigma=(1,0)$ is an ESS of level $k$ if and only if $\beta_{0}=\beta_{1}=\cdots=\beta_{k-1}=0$ and $\beta_{k}>0$. In this case the lowest order term in (3) is $C_{j}^{n-1} \delta^{k+1} \beta_{k}>0$ and so for small $\delta$, i.e., $x \approx \sigma$, we have that $\Pi(x)>0$ and so $\sigma=(1,0)$ is an LSS. Similarly, one may prove that if $\sigma=(0,1)$ is an ESS then it is an LSS.

Now consider a mixed ESS $\sigma=\left(\sigma_{1}, 1-\sigma_{1}\right)$ with $0<\sigma_{1}<1$. Consider $x=$ $\left(x_{1}, 1-x_{1}\right)$. Define

$$
h(t)=\sum_{i=0}^{n-1} C_{i}^{n-1} t^{n-1-i} \beta_{i}
$$

where $t=\frac{x_{1}}{1-x_{1}}$. After some manipulations we get

$$
\Pi(x)=\left(\sigma_{1}-x_{1}\right)\left(1-x_{1}\right)^{n-1} h(t) .
$$

In Bromm et al. [1997] it is proven that a mixed ESS of level $k$ with $k$ being odd satisfies $h(t)=0, h^{(j)}(t)=0$ for all $j<k$ and $h^{(k)}(t)<0$ at $t=\frac{\sigma_{1}}{1-\sigma_{1}}$ where $h^{(j)}$ 
is the $j$ th-order derivative of $h$, and that there are no mixed ESS of level $k$ when $k$ is even. This implies that $\Pi$ and all the derivatives of $\Pi$ up to order $k$ at $x=\sigma$ vanish. Furthermore, the derivative

$$
\left.\Pi^{(k+1)}(x)\right|_{x=\sigma}=-k\left(1-\sigma_{1}\right)^{n-1} h^{(k)}(t)>0 .
$$

Since $k+1$ is even this proves that $\Pi(x)>0$ for $x \approx \sigma$ and so $\sigma$ is an LSS.

We conclude the following:

Theorem 1. The concepts of ESS, ESS with uniform invasion barrier and LSS are equivalent for every symmetric games with two players and for every $n$-player game with two pure strategies.

However, when the number of players and the number of pure strategies are not 2, there may exist ESSs that are not locally superior, or in other words, ESSs that have no uniform invasion barrier. We provide an example.

Example 4. Consider a four-player, three-strategy game. Denote the three pure strategies by 1,2 and 3 , respectively. Denote the payoffs to player 1 by $a_{i, j, k, l}$ where $i, j, k, l \in\{1,2,3\}$ are the pure strategy choices of players $1-4$. By symmetry, $a_{i, j, k, l}=a_{i, \sigma(i), \sigma(j), \sigma(k)}$ where $\sigma$ is a permutation of three elements. Consider the following payoffs:

$$
\begin{aligned}
& a_{3,3,1,1}=-3, \\
& a_{2,2,2,2}=-2, \\
& a_{3,2,2,1}=10 .
\end{aligned}
$$

Consider the remaining payoffs equal to zero, except the ones defined by symmetry, such as $a_{3,1,3,1}=a_{3,1,1,3}=-3$ and $a_{3,2,2,1}=a_{3,2,1,2}=a_{3,1,2,2}=10$. Denote $\sigma=(1,0,0)$. We have $\pi(\sigma, \sigma, \sigma)=0=\pi(\mu, \sigma, \sigma)$ for any $\mu=\left(\mu_{1}, \mu_{2}, \mu_{3}\right) \in \Delta S$. Furthermore, $\pi(\mu, \mu, \sigma, \sigma)=-3 \mu_{3}^{2} \leq \pi(\sigma, \mu, \sigma, \sigma)=0$, with equality if and only if $\mu_{3}=0$. Now if $\mu_{3}=0$ we have $\pi(\sigma, \mu, \mu, \sigma)=0=\pi(\mu, \mu, \mu \sigma)$ and $\pi(\mu, \mu, \mu, \mu)=$ $-2 \mu_{2}^{4} \leq \pi(\sigma, \mu, \mu, \mu)$ with equality if and only if $\mu_{2}=0$, which, since $\mu_{3}=0$, means that $\mu=\sigma$. So $\sigma$ is an ESS by Proposition 1 .

Now $\pi(\sigma, x, x, x)=0$ and $\pi(x, x, x, x)=-9 x_{1}^{2} x_{3}^{2}-2 x_{2}^{4}+30 x_{1} x_{2}^{2} x_{3}$. Taking into account that $x_{1}+x_{2}+x_{3}=1$ and putting $x_{3}=\left(1-x_{1}\right)^{2}$ we obtain that $\pi(x, x, x, x)=-\left(x_{1}-1\right)^{4} x_{1}^{2}\left(2 x_{1}^{2}-30 x_{1}+9\right)$. When $x_{1} \lesssim 1$ we have that $0<$ $\pi(x, x, x, x)$ and so we have a neighborhood of $\sigma$ where (2) does not hold and so $\sigma$ is not locally superior.

\subsection{The ESS set is finite}

In this subsection we show that the set of ESSs is a finite set. To do this, we prove that ESSs are locally unique. 
We shall start by proving the following auxiliary lemmas:

Lemma 2. For $n \geq 1$ we have the following identity:

$$
\pi(z, \underbrace{a x+b y, \ldots, a x+b y}_{(n)})=\sum_{k=0}^{n} C_{k}^{n} a^{n-k} b^{k} \pi(z, \underbrace{x, \ldots, x}_{(n-k)}, \underbrace{y, \ldots, y}_{(k)}) .
$$

Proof. We proceed by induction. The equality is obvious for $n=1$. Now assume that the equality holds for $h \leq n-1$. We have

$$
\begin{aligned}
\pi(z, a x+b y, \underbrace{a x+b y, \ldots, a x+b y}_{(n-1)}) \\
=\sum_{k=0}^{n-1} C_{k}^{n-1} a^{n-k-1} b^{k} \pi(z, a x+b y, \underbrace{x, \ldots, x}_{(n-k-1)}, \underbrace{y, \ldots, y}_{(k)}) .
\end{aligned}
$$

Now expanding and rearranging the terms and taking into account the symmetry of the game and the equality

$$
C_{k}^{n-1}+C_{k+1}^{n-1}=C_{k+1}^{n}
$$

the claim follows.

Remark 3. Let $\boldsymbol{\sigma}$ be a Symmetric Nash Equilibrium profile and let $\left\{\boldsymbol{\sigma}^{\epsilon}\right\}_{\epsilon \rightarrow 0}$ be a sequence of Symmetric Nash Equilibrium profiles such that $\boldsymbol{\sigma}^{\epsilon} \rightarrow \boldsymbol{\sigma}$, when $\epsilon \rightarrow$ 0 . There exists $\epsilon_{0}$ sufficiently small such that for all $\epsilon<\epsilon_{0}, C(\sigma) \subseteq C\left(\sigma^{\epsilon}\right) \subseteq$ $B\left(\sigma^{\epsilon}, \ldots, \sigma^{\epsilon}\right) \subseteq B(\sigma, \ldots, \sigma)$ and it follows that

$$
\begin{aligned}
\pi\left(\sigma, \sigma^{\epsilon}, \ldots, \sigma^{\epsilon}\right) & =\pi\left(\sigma^{\epsilon}, \sigma^{\epsilon}, \ldots, \sigma^{\epsilon}\right) \quad \text { and } \quad C(\sigma) \subseteq B\left(\boldsymbol{\sigma}^{\epsilon}\right), \\
\pi\left(\sigma^{\epsilon}, \sigma, \ldots, \sigma\right) & =\pi(\sigma, \sigma, \ldots, \sigma) \text { and } C\left(\sigma^{\epsilon}\right) \subseteq B(\boldsymbol{\sigma}) .
\end{aligned}
$$

Lemma 3. Let $\sigma^{\epsilon}$ be a sequence of mixed strategies such that $\sigma^{\epsilon} \rightarrow \sigma$, when $\epsilon \rightarrow 0$, with $\sigma^{\epsilon} \neq \sigma$, we define $\delta(\epsilon)=\max _{j}\left\{\frac{\left|\sigma_{j}-\sigma_{j}^{\epsilon}\right|}{\sigma_{j}}: \sigma_{j}>0\right\}$. Then:

(1) $\delta(\epsilon) \rightarrow 0$ when $\epsilon \rightarrow 0$;

(2) $\mu^{\epsilon}=\frac{\sigma^{\epsilon}-(1-\delta(\epsilon)) \sigma}{\delta(\epsilon)} \in \Delta(S)$ and $\sigma^{\epsilon}=(1-\delta(\epsilon)) \sigma+\delta(\epsilon) \mu^{\epsilon}$.

Proof. (1) Clear since $\sigma^{\epsilon} \rightarrow \sigma$ when $\epsilon \rightarrow 0$.

(2) We first prove that $\mu_{j}^{\epsilon} \geq 0$. If $\sigma_{j}=0$ we have that $\mu_{j}^{\epsilon}=\frac{\sigma_{j}^{\epsilon}}{\delta(\epsilon)} \geq 0$ because $\delta(\epsilon)>0$ and $\sigma^{\epsilon} \in \Delta(S)$, i.e., $\sigma_{j}^{\epsilon} \geq 0$. If $\sigma_{j}>0, \mu_{j}^{\epsilon}=\frac{\sigma_{j}^{\epsilon}-(1-\delta(\epsilon)) \sigma_{j}}{\delta(\epsilon)} \geq 0$ is equivalent to $\frac{\sigma_{j}-\sigma_{j}^{\epsilon}}{\sigma_{j}} \leq \delta(\epsilon)$ which is true by definition of $\delta(\epsilon)$.

It remains to prove that $\sum_{j=1}^{n} \mu_{j}^{\epsilon}=1$. By definition we have that $\sum_{j=1}^{n} \mu_{j}^{\epsilon}=$ $\frac{1}{\delta(\epsilon)} \sum_{j=1}^{n}\left(\sigma_{j}^{\epsilon}-(1-\delta(\epsilon)) \sigma_{j}\right)=\frac{1}{\delta(\epsilon)}(1-(1-\delta(\epsilon)))=1$. This proves that $\mu^{\epsilon} \in \Delta(S)$. 
Lemma 4. Let $\boldsymbol{\sigma}$ be ESS and let $\boldsymbol{\sigma}^{\epsilon}$ be a sequence of ESSs such that $\boldsymbol{\sigma}^{\epsilon} \rightarrow \boldsymbol{\sigma}$, when $\epsilon \rightarrow 0$, where, for all $\epsilon>0$, we have that $\boldsymbol{\sigma}^{\epsilon} \neq \boldsymbol{\sigma}$. If $\pi\left(\sigma^{\epsilon}, \sigma, \sigma^{\epsilon}, \ldots, \sigma^{\epsilon}\right) \geq$ $\pi\left(\sigma, \sigma, \sigma^{\epsilon}, \ldots, \sigma^{\epsilon}\right)$ and there exists $k^{\prime} \geq 0$, such that for all $0 \leq k \leq k^{\prime}$,

$$
\pi(\mu^{\epsilon}, \sigma, \underbrace{\sigma, \ldots, \sigma}_{(n-2-k) \text {-times }}, \underbrace{\mu^{\epsilon}, \ldots, \mu^{\epsilon}}_{k \text {-times }})=\pi(\sigma, \sigma, \underbrace{\sigma, \ldots, \sigma}_{(n-2-k) \text {-times }}, \underbrace{\mu^{\epsilon}, \ldots, \mu^{\epsilon}}_{k \text {-times }}),
$$

where $\mu^{\epsilon}$ and $\delta(\epsilon)$ are as in Lemma 3 , then

$$
\pi(\mu^{\epsilon}, \sigma, \underbrace{\sigma, \ldots, \sigma}_{\left(n-3-k^{\prime}\right) \text {-times }\left(k^{\prime}+1\right) \text {-times }}, \underbrace{\mu^{\epsilon}, \ldots, \mu^{\epsilon}}_{\left(n-3-k^{\prime}\right) \text {-times }\left(k^{\prime}+1\right) \text {-times }}) \geq \pi(\sigma, \sigma, \underbrace{\mu^{\epsilon}, \ldots, \mu^{\epsilon}}_{(.,}) .
$$

Proof. Let $\boldsymbol{\sigma}$ be an ESS and let $\sigma^{\epsilon}$ be a sequence of ESSs such that $\sigma^{\epsilon} \rightarrow \sigma$, when $\epsilon \rightarrow 0$, and $\sigma^{\epsilon} \neq \sigma$. Let $\mu^{\epsilon}, \delta(\epsilon)$ be as in Lemma 3. Since $\sigma^{\epsilon}=(1-\delta(\epsilon)) \sigma+\delta(\epsilon) \mu^{\epsilon}$ and by hypothesis $\pi\left(\sigma^{\epsilon}, \sigma, \sigma^{\epsilon}, \ldots, \sigma^{\epsilon}\right) \geq \pi\left(\sigma, \sigma, \sigma^{\epsilon}, \ldots, \sigma^{\epsilon}\right)$, we have that

$$
(1-\delta(\epsilon)) \pi\left(\sigma, \sigma, \sigma^{\epsilon}, \ldots, \sigma^{\epsilon}\right)+\delta(\epsilon) \pi\left(\mu^{\epsilon}, \sigma, \sigma^{\epsilon}, \ldots, \sigma^{\epsilon}\right) \geq \pi\left(\sigma, \sigma, \sigma^{\epsilon}, \ldots, \sigma^{\epsilon}\right),
$$

this implies that $\pi\left(\mu^{\epsilon}, \sigma, \sigma^{\epsilon}, \ldots, \sigma^{\epsilon}\right) \geq \pi\left(\sigma, \sigma, \sigma^{\epsilon}, \ldots, \sigma^{\epsilon}\right)$. After some algebra it holds that

$$
\begin{aligned}
\pi\left(\mu^{\epsilon},\right. & \left.\sigma, \sigma^{\epsilon}, \ldots, \sigma^{\epsilon}\right) \\
& =\sum_{k=0}^{n-2}\left(\begin{array}{c}
n-2-k \\
k
\end{array}\right)(1-\delta(\epsilon))^{n-2-k} \delta(\epsilon)^{k} \pi(\mu^{\epsilon}, \sigma, \underbrace{\sigma, \ldots, \sigma}_{(n-2-k) \text {-times }}, \underbrace{\mu^{\epsilon}, \ldots, \mu^{\epsilon}}_{k \text {-times }}) \\
& \geq \pi\left(\sigma, \sigma, \sigma^{\epsilon}, \ldots, \sigma^{\epsilon}\right) \\
& =\sum_{k=0}^{n-2}\left(\begin{array}{c}
n-2-k \\
k
\end{array}\right)(1-\delta(\epsilon))^{n-2-k} \delta(\epsilon)^{k} \pi(\sigma, \sigma, \underbrace{\sigma, \ldots, \sigma}_{(n-2-k) \text {-times }}, \underbrace{\mu^{\epsilon}, \ldots, \mu^{\epsilon}}_{k \text {-times }}) .
\end{aligned}
$$

By assumption there exists $k^{\prime} \geq 0$, such that for all $0 \leq k \leq k^{\prime}$,

$$
\pi(\mu^{\epsilon}, \sigma, \underbrace{\sigma, \ldots, \sigma}_{(n-2-k) \text {-times }}, \underbrace{\mu^{\epsilon}, \ldots, \mu^{\epsilon}}_{k \text {-times }})=\pi(\sigma, \sigma, \underbrace{\sigma, \ldots, \sigma}_{(n-2-k) \text {-times }}, \underbrace{\mu^{\epsilon}, \ldots, \mu^{\epsilon}}_{k \text {-times }})
$$

and $\delta(\epsilon) \rightarrow 0$, then for $\epsilon$ sufficiently small we have that

$$
\pi(\mu^{\epsilon}, \sigma, \underbrace{\sigma, \ldots, \sigma}_{\left(n-3-k^{\prime}\right) \text {-times }}, \underbrace{\mu^{\epsilon}, \ldots, \mu^{\epsilon}}_{\left(k^{\prime}+1\right) \text {-times }}) \geq \pi(\sigma, \sigma, \underbrace{\sigma, \ldots, \sigma}_{\left(n-3-k^{\prime}\right) \text {-times }}, \underbrace{\mu^{\epsilon}, \ldots, \mu^{\epsilon}}_{\left(k^{\prime}+1\right) \text {-times }}),
$$

which proves the lemma. 
Proposition 3. If $\sigma$ is an ESS then $\sigma$ is isolated within ESS.

Proof. If not, there exists an ESS sequence $\sigma^{\epsilon} \rightarrow \sigma$, when $\epsilon \rightarrow 0$ and $\sigma^{\epsilon} \neq \sigma$. Since $\sigma$ and $\sigma^{\epsilon}$ are ESSs we have that

$$
\pi(\sigma, \ldots, \sigma) \geq \pi\left(\sigma^{\epsilon}, \sigma, \ldots, \sigma\right)
$$

and

$$
\pi\left(\sigma^{\epsilon}, \ldots, \sigma^{\epsilon}\right) \geq \pi\left(\sigma, \sigma^{\epsilon}, \ldots, \sigma^{\epsilon}\right) .
$$

By Remark 3 we have that previous inequalities are, in fact, equalities, i.e.,

$$
\pi(\sigma, \ldots, \sigma)=\pi\left(\sigma^{\epsilon}, \sigma, \ldots, \sigma\right)
$$

and

$$
\pi\left(\sigma^{\epsilon}, \ldots, \sigma^{\epsilon}\right)=\pi\left(\sigma, \sigma^{\epsilon}, \ldots, \sigma^{\epsilon}\right) .
$$

From condition (5), Lemma 3 and $\sigma^{\epsilon}=(1-\delta(\epsilon)) \sigma+\delta(\epsilon) \mu^{\epsilon}$ we have that

$$
\pi(\sigma, \ldots, \sigma)=\pi\left(\mu^{\epsilon}, \sigma, \ldots, \sigma\right) .
$$

Let

$$
\begin{aligned}
k^{\prime} & =\max \left\{t: \forall 0 \leq k \leq t, \pi(\mu^{\epsilon}, \sigma, \underbrace{\sigma, \ldots, \sigma}_{(n-2-k)}, \underbrace{\mu^{\epsilon}, \ldots, \mu^{\epsilon}}_{k})\right. \\
& \left.=\pi(\sigma, \sigma, \underbrace{\sigma, \ldots, \sigma}_{(n-2-k)}, \underbrace{\mu^{\epsilon}, \ldots, \mu^{\epsilon}}_{k})\right\},
\end{aligned}
$$

from the equality (7) it follows that $k^{\prime} \geq 0$.

Now, since for all $0 \leq k \leq k^{\prime}$,

$$
\pi(\mu^{\epsilon}, \sigma, \underbrace{\sigma, \ldots, \sigma}_{(n-2-k) \text {-times }}, \underbrace{\mu^{\epsilon}, \ldots, \mu^{\epsilon}}_{k \text {-times }})=\pi(\sigma, \sigma, \underbrace{\sigma, \ldots, \sigma}_{(n-2-k) \text {-times }}, \underbrace{\mu^{\epsilon}, \ldots, \mu^{\epsilon}}_{k \text {-times }}),
$$

from Lemma 4 and the definition of $k^{\prime}$, it implies that

$$
\pi(\mu^{\epsilon}, \sigma, \underbrace{\sigma, \ldots, \sigma}_{\left(k^{\prime}+1\right) \text {-times }}, \underbrace{\mu^{\epsilon}, \ldots, \mu^{\epsilon}}_{\left(n-3-k^{\prime}\right) \text {-times }})>\pi(\sigma, \sigma, \underbrace{\sigma, \ldots, \sigma}_{\left(k^{\prime}+1\right) \text {-times }}, \underbrace{\mu^{\epsilon}, \ldots, \mu^{\epsilon}}_{\left(n-3-k^{\prime}\right) \text {-times }}),
$$

contradicting that $\sigma \in E S S$. Then $\sigma$ is an isolated ESS. 


\section{The Extended Replicator Dynamics}

The aim of this section is to study the relationship between asymptotic stability and ESS in the dynamics of the replicator for the case of multi-population symmetric games. We show some particular results for these games. Finally, in order to understand the evolution of investor decisions in financial assets, we present an application to the theory of portfolios.

As in Weibull [1995] we consider a $K$-population symmetric game, $K \geq 1$, where each individual in each population can choose one of $m$ different behaviors or pure strategies. As usual, the replicator dynamics assumes that the populations have a large number of individuals (technically infinite).

We say that an individual is an $i$ th strategist if he chooses the pure strategy $i \in$ $\{1, \ldots, m\}$. In this way each population is divided into $m$ different subpopulations, according to the strategy followed by the individuals. As usual in population games we may think of a mixed strategy also as a state of the populations. Suppose that the composition of the population $p$ is described by the mixed strategy, or state $x=\left(x_{1}, \ldots, x_{K}\right)$, a mixed strategy profile, where $x_{p}=\left(x_{p 1}, \ldots, x_{p m}\right)$ denotes the probability that an individual in population $p$ is playing each one of its pure strategies. Then obviously for all $p \in\{1, \ldots, K\}$ we have that $\sum_{i=1}^{m} x_{p i}=1$ and $0 \leq x_{p i} \leq 0$ for all $i \in\{1, \ldots, m\}$.

For $p \neq l$ let $R_{i}^{l}\left(e_{p}^{j_{p}}\right)=R_{i}^{l}\left(e_{1}^{j_{1}}, \ldots, e_{l-1}^{j_{l-1}}, e_{l+1}^{j_{l+1}}, \ldots, e_{K}^{j_{K}}\right)$ be the payoff for a $i$ th strategist in population $l$ when the other populations choose strategy $e_{p}^{j_{p}}$, for each $p \neq l$. Given the symmetry of the game we have that $R_{i}^{l}\left(e_{p}^{j_{p}}\right)=R_{i}^{l}\left(s\left(e_{p}^{j_{p}}\right)\right)$ where $s$ is any permutation on $p-1$ symbols.

Let $x=\left(x_{1}, \ldots, x_{k}\right) \in \mathbb{R}^{K m}$ be a vector whose coordinates indicate a distribution over the set of pure strategies in each population and denote the distribution in population other than $l$ by $x_{-l}=\left(x_{1}, \ldots, x_{l-1}, x_{l+1}, \ldots, x_{K}\right)$.

The expected payoff of a player in population $l$ playing according to the $i$ th pure strategy given the population state $x$ is

$$
E\left(e^{i} \mid x_{-l}\right)=\sum_{j_{p}=1, p=1, \ldots, K, p \neq l}^{m} R_{i}^{l}\left(e_{1}^{j_{1}}, \ldots, e_{l-1}^{j_{l-1}}, e_{l+1}^{j_{l+1}}, \ldots, e_{K}^{j_{K}}\right) P_{i}\left(x_{-l}\right),
$$

where the sum is over choice of pure strategies in the other populations and $P_{i}\left(x_{-l}\right)=\Pi_{p \neq l} x_{p j_{p}}=x_{1 j_{1}} \cdots x_{l-1, j_{l-1}} x_{l+1, j_{l+1}} \cdots x_{K, j_{K}}$ are the probabilities of each choice according to the population distribution $x_{-l}$.

The expected value for a population playing according to a distribution $x_{l}$ given the distribution $x_{-l}$ for the other players is given by

$$
E\left(x_{l}, x_{-l}\right)=\sum_{i=1}^{m} x_{l i} E\left(e^{i} \mid x_{-l}\right) .
$$

The replicator dynamics is then given by the following system of ordinary differential equations:

$$
\dot{x}_{l i}=\left[E\left(e^{i}, x_{-l}\right)-E\left(x_{l}, x_{-l}\right)\right] x_{l i}, \quad l=1, \ldots, k \quad \text { and } \quad i=1, \ldots, m .
$$


Note that if the expected value for the $i$ th strategist in population $l$ given a $h_{l}$ is bigger than the average payoff in the population, then the percentage of individuals following this strategy increases with time. Since $E\left(x_{l}, x_{-l}\right)=\sum_{i=1}^{m} x_{l i} E\left(e^{i}, x_{-l}\right)$ and $\sum_{i=1}^{m} x_{l i}=1$ the system is invariant in the $K$-dimensional simplex. Furthermore, we have that any point where every player is playing according to one of its pure strategies is a dynamic equilibrium for the game, i.e., the vertexes of the simplex are equilibrium points. Also, we have that any Symmetrical Nash Equilibrium of the game is also a dynamic equilibrium, but the converse is not necessarily true. The converse holds if the dynamic equilibrium lies in the interior of the simplex.

\subsection{Dynamic stability and ESS}

Unlike the classic $n=2$ replicator dynamics, in multi-population replicator dynamics a locally superior strategy (and consequently an ESS) is not necessarily an asymptotically stable point. We show an example of an LSS that is not asymptotically stable. Indeed, following the results in Weibull [1995] the asymptotically stable states are the Strict Nash Equilibria which, as we mentioned, is equivalent to the general ESS definition. Using one of our previous examples of an ESS that is not a Strict Nash we obtain an example showing that the $n=2$ classic results do not extend to the ESS definition considered here for $n \geq 3$.

Example 5. Consider the three-player two-strategy with the following payoff matrices (as in Example 31):

$$
\left[\begin{array}{ll}
1 & 1 \\
1 & 1
\end{array}\right] \quad\left[\begin{array}{ll}
1 & 1 \\
1 & 0
\end{array}\right] .
$$

Assume that player 1 is following the strategy $x=\left(x_{1}, x_{2}\right)$, player 2 the strategy $y=\left(y_{1}, y_{2}\right)$ and player 3 the strategy $z=\left(z_{1}, z_{2}\right)$. Then the replicator equation for the first population takes the form

$$
\dot{x}_{1}=x_{1}\left[E_{1}\left(e^{1}, y, z\right)-E_{1}(x, y, z)\right]=x_{1}\left(1-x_{1}\right)\left[\left(y_{1}-1\right)\left(z_{1}-1\right)\right] .
$$

Given that $x_{1}+x_{2}=1$ it follows that

$$
\dot{x}_{2}=-\dot{x_{1}} \text {. }
$$

Analogously for the second player we have that

$$
\begin{aligned}
& \dot{y}_{1}=y_{1}\left(1-y_{1}\right)\left[E_{2}\left(e^{1}, x, z\right)-E_{2}\left(e^{2}, x, z\right)\right]=y_{1}\left(1-y_{1}\right)\left[\left(x_{1}-1\right)\left(z_{1}-1\right)\right], \\
& \dot{y}_{2}=-\dot{y}_{1} .
\end{aligned}
$$

For the third player we have

$$
\begin{aligned}
& \dot{z}_{1}=z_{1}\left(1-z_{1}\right)\left[E_{3}\left(e^{1}, x, y\right)-E_{3}\left(e^{2}, x, y\right)\right]=z_{1}\left(1-z_{1}\right)\left[\left(x_{1}-1\right)\left(y_{1}-1\right)\right], \\
& \dot{z}_{2}=-\dot{z}_{1}
\end{aligned}
$$


The point $\phi^{*}=(1,0,1,0,1,0)$ is a dynamical equilibrium for the system. On the other hand, as we have seen in Example 3 this point corresponds to a Nash Equilibrium strategic profile and moreover it is an ESS which is also an LSS by Proposition 2 since there are only two pure strategies. Despite having these properties, this point is not a Strict Nash Equilibrium since for every $z \in[0,1]$ the point $\phi=(1,0,1,0, z, 1-z)$ is a dynamical equilibrium for the system. So, in every neighborhood of $(1,0,1,0,1,0)$ there exists a point $(1,0,1,0, z, 1-z)$ such that trajectories starting at it do not approach $(1,0,1,0,1,0)$. Hence $(1,0,1,0,1,0)$ is not asymptotically stable.

\subsection{Application}

Suppose that $K$ investors, indexed by $p \in\{1, \ldots, K\}$, need to choose at the same time how much money to invest in the same $m$ different alternatives. Let us denote these alternatives by $T_{1}, \ldots, T_{m}$. Assume that each investor has the same amount of money, suppose that the cost to invest in each alternative is the same, then $n_{p}=n_{p T_{1}}+\cdots+n_{p T_{m}}=n$ for all $p=1, \ldots, K$, where $n_{p T_{i}}$ is the amount of alternatives $T_{i}$ possessed by the $p$ th investor. According to the financial literature $n_{p}$ corresponds to a portfolio of alternatives chosen by the $p$ th investor which describes the amount that the investor chooses for each one of the possible alternatives.

A mixed strategy for the $p$ th investor will be denoted by $x_{p}=\left(x_{p T_{1}}, \ldots, x_{p T_{m}}\right)$ where $x_{p T_{i}}=n_{p T_{i}} / n$ corresponds to the percentage of asset $T_{i}$ in the portfolio of the $p$ th investor, for all $p \in\{1, \ldots, K\}, k \in\{1, \ldots, m\}$. By $\mathbf{x}=\left(x_{1}, \ldots, x_{K}\right)$ we denote a mixed strategy profile. Note that $x_{p} \in \Delta^{m-1}$ where by $\Delta^{m-1}$ we symbolize the $(m-1)$-dimensional simplex. Consequently,

$$
\mathbf{x} \in \underbrace{\Delta^{m-1} \times \cdots \times \Delta^{m-1}}_{K}=\left(\Delta^{m-1}\right)^{K}
$$

By

$$
R^{l}\left(T_{i}, T_{-l}\right)=A^{l T_{l}}(\underbrace{T_{1}, \ldots, T_{1}}_{K-1}), \ldots, A^{l T_{i}}(\underbrace{T_{i_{1}}, \ldots, T_{i_{K}}}_{K-1}), \ldots, A^{l T_{i}}(\underbrace{T_{m}, \ldots, T_{m}}_{K-1}),
$$

we denote the payoffs corresponding to the $l$ th investor when he is playing according to the pure strategy $T_{i}$ and each one of the others is choosing one alternative in the set $\left(T_{1}, \ldots, T_{m}\right)$. Note that each term $\underbrace{T_{i_{1}}, \ldots, T_{i_{k}}}_{K-1}$ corresponds to one of the variations with repetitions $V_{K-1}^{m}$ of the set of alternatives $\left(T_{1}, \ldots, T_{m}\right)$, the total amount of these possibilities is $\left|V_{K-1}^{m}\right|=(K-1)^{m}$.

The payoff corresponding to each investor depends on the distribution of investments of the other investors over the set of possible alternatives, $n_{-l}=$ $\left(n_{1}, \ldots, x_{l-1}, n_{l+1}, \ldots, n_{K}\right)$. 
The expected payoff corresponding to the alternative $T_{i}$ for the $l$ th investor when the others are investing according to

$$
x_{-l}=\left(x_{1}, \ldots, x_{l-1}, x_{l+1}, \ldots, x_{m}\right),
$$

is denoted by the expression

$$
E^{l}\left(T_{i}, x_{-l}\right)=\sum_{V_{K-1}^{m}}[A^{l T_{i}} \underbrace{\left(T_{j_{1}}, \ldots, T_{j_{K}}\right)}_{K-1} \Pi_{i \neq l} x_{i T_{j_{i}}}],
$$

where $A^{l T_{i}}(\underbrace{T_{j_{1}} \ldots T_{j_{K}}}_{K-1})$ denotes the payoff obtained by the $l$ th investor when he is playing according to his pure strategy $T_{i}$ and the others are choosing

$$
T_{-l}=\left(T_{j_{1}}, \ldots, T_{j_{l-1}}, T_{j_{l+1}}, \ldots, T_{j_{K}}\right)
$$

and $\Pi_{i \neq l} x_{i T_{j_{i}}}=x_{1 T_{1}} \ldots x_{l-1, T_{l-1}}, x_{l+1, T_{l+1}} \ldots x_{K T_{K}}$.

Then the expected payoff for the $l$ th investor when he is choosing a portfolio $n_{l}=\left(n_{l T_{1}}, \ldots, n_{l T_{K}}\right)$, given that the other investors are choosing $n_{-l}$, is given by

$$
E\left(x_{l}, x_{-l}\right)=\sum_{j=1}^{m} \frac{n_{l T_{j}}}{n} E^{l}\left(T_{j}, n_{-l}\right)=\sum_{j=1}^{m} x_{l T_{j}} E^{l}\left(T_{j}, x_{-l}\right) .
$$

Remark 4. Let it be noticed that by the symmetry of the game we have that the expected value for each investor does not change due to rearrangements in the investment portfolios, i.e.,

$$
E\left(x_{1}, \ldots, x_{K}\right)=E\left[s\left(x_{1}, \ldots, x_{K}\right)\right],
$$

where $s\left(x_{1}, \ldots, x_{K}\right)$ is a permutation of the coordinates of the vector

$$
\mathbf{x}=\left(x_{1}, \ldots, x_{K}\right) \in\left(\Re^{m}\right)^{K} .
$$

\subsection{The dynamics of the investment}

Our interest now is to analyze the changes in the distribution of the alternatives along the time. We assume that every investor looks for him the best strategy taking account the set of strategies followed by his competitors.

Suppose that at the end of every period, after that profits of the investments are revealed, each investor must choose the amount to invest in each alternative for the next period, i.e., at the end of each period, each investor must choose a new portfolio for the next period, or equivalently a mixed strategy.

Given that for all $p \in\{1, \ldots, K\}, x_{p}=\left(x_{p T_{1}}, \ldots, x_{p T_{m}}\right)=\left(\frac{n_{p T_{1}}}{n}, \ldots, \frac{n_{p T_{m}}}{n}\right)$, the replicator dynamics for this game will be given by the dynamical system with $m K$ differential equations,

$$
\dot{x}_{p T_{j}}=x_{p T_{j}}\left[E^{p}\left(T_{j}, x_{-p}\right)-E\left(x_{p}, x_{-p}\right)\right], \quad p=1, \ldots, K \quad \text { and } \quad j=1, \ldots, m .
$$


Note that if the mixed profile strategy $\mathbf{x}^{*}=\left(x_{1}^{*}, \ldots, x^{K}\right)$ is a Nash Equilibrium then $E^{p}\left(T_{j}, x_{-p}^{*}\right)=E\left(x_{p}^{*}, x_{-p}^{*}\right)$ for all $j \in\{1, \ldots, m\}$ and $p \in\{1, \ldots, K\}$. It is easy to see that the strategy profile $\mathbf{x}^{*}$ is also a dynamical equilibrium for the replicator dynamics. The reciprocal is verified only partially, i.e., if the strategy profile $\mathbf{x}^{*}$ is interior to $\left(\Delta^{m-1}\right)^{K}$ then $\mathbf{x}^{*}$ is also a Nash Equilibrium.

Given the symmetry of our model note that if the profile $\mathbf{x}^{*}$ is a Nash Equilibrium, then $x_{1}^{*}=\cdots=x_{K}^{*}$.

The claim that the investors are plying according to the Nash Equilibrium $\mathbf{x}^{*}$ means that each investor is making the best individual choice of investment given the choices of the others, i.e., $n_{p}^{*}$ is the best choice of the $p$ th investor given that the others are investing the amounts $n_{-p T_{j}}^{*}, p \in\{1, \ldots, K\}$, in each alternative $T_{j}, j \in\{1, \ldots, m\}$. Or equivalently, $E^{p}\left(x_{p}^{*}, x_{-p}^{*}\right) \geq E^{p}\left(x_{p}, x_{-p}^{*}\right)$ for all $x_{p} \in \Delta^{m-1}$ and $p=1, \ldots, K$.

Moreover, if $\mathbf{x}^{*}$ is a Nash Equilibrium, all the investors are investing the same share in the same alternatives. For the question of whether to maintain the strategy given that $\mathrm{x}^{*}$ continues being a best response, when some individuals change their investment it is natural, and the answer is in the definition of Evolutionarily Stable Strategy, see Definition 2 and its dynamic properties. Note that if $\mathbf{x}^{*}$ is an ESS, keeping the original choice is a best response, even when the investors change their choices, as long as this change is not very large.

Given that all investors in this application are identical, then the probability to change the strategy that they followed until some time $t$ with another fixed strategy is the same for everyone.

If in addition this equilibrium is asymptotically stable for the replicator dynamics (see Sec. 4.1), with the passage of time, those investors who made the decision to change the initial strategy (specified in $\mathbf{x}^{*}$ ) will return to the initial position.

\section{Conclusions}

In this paper, we considered a definition of ESS for multi-population symmetric games differing from the general definition of ESS not necessarily for symmetric games which is equivalent to the notion of Strict Nash Equilibrium. We analyzed some properties of the definition and provided some examples of specific games to illustrate the results. We showed that the set of ESS is finite. Furthermore, unlike the case of games that have only two players some alternative characterizations of ESS do not hold, such as the property of being locally superior, i.e., performing better against neighboring strategies than the neighbors against themselves. This property is known to be equivalent to the existence of a uniform invasion barrier for the ESS, i.e., a maximal mutation size that works for every mutation uniformly. We provide an example showing that this characterization of ESS does not hold in general and so the invasion barrier of such ESS is not necessarily uniform, i.e., for some specific mutation the maximum invading size must be smaller than for others. Nevertheless, we showed that regardless of the number of players, for two-strategy games, the 
original characterization still holds. Finally, we considered the replicator dynamics and showed that a classical result for two-player games, namely the stability of an ESS, no longer holds. We end with an application of the replicator dynamics.

\section{Acknowledgments}

Elvio Accinelli is grateful to CONACYT for the research support by means of the Project 167004 and to LIAAD-INESC TEC for the support through the Project Dynamics (PTDC/MAT-NAN/6890/2014).

Jorge Oviedo is grateful to Universidad Nacional de San Luis, for the support through the Grant 03-2016, and to the Consejo Nacional de Investigaciones Científicas y Técnicas (CONICET), for the support through the Grant PIP 112200801-00655.

\section{References}

Accinelli, E. and Carrera, E., [2011] Evolutionarily stable strategies and replicator dynamics in asymmetric two-population games, in Dynamics, Games and Science I, in Honour of Maurício Peixoto and David Rand, eds. Peixoto, M. Pinto A. \& Rancl, D., Springer Proceedings in Mathematics, Vol. 1 (Springer, Berlin), pp. 25-35.

Accinelli, E., Bazzano, B., Robledo, F., and Romero, P. [2015] Nash equilibrium in evolutionary competitive models of firms and workers, J. Dyn. Games 2, 1-32.

Bromm, M., Cannings, C. and Vickers, G. T. [1997] Multi-player matrix games, Bull. Math. Bio. 59, 931-952.

Bukowski, M. and Miekisz, J. [2004] Evolutionary and asymptotic stability in symmetric multi-player games, Int. J. Game Theory 33, 41-54.

Gokhale, C. S. and Traulsen, A. [2010] Evolutionary games in the multiverse, Proc. Natl. Acad. Sci. 107, 5500-5504.

Kurokawa, S. and Ihara, Y. [2009] Emergence of cooperation in public goods games, Proc. R. Soc. B. Biol. Sci. 276, 1379-1384.

Maynard Smith, J. [1982] Evolution and the Theory of Games (Cambridge University Press, Cambridge).

Maynard Smith, J. and Price, G. R., [1973] The logic of animal conflict, Nature 246, $15-18$.

Nash, J. F. [1951] Non-cooperative games, Ann. Math. 54 286-295.

Palm, G. [1984] Evolutionary stable strategies and game dynamics for $n$-person games, $J$. Math. Bio. 19, 329-334.

Taylor, P. [1979] Evolutionarily stable strategies with two types of player, J. Appl. Probab. 16, 76-83.

van Damme, E. [1987] Stability and Perfection of Nash Equilibria (Springer-Verlag, Berlin).

van Veelen, M. [2009] Group selection, kin selection, altruism and cooperation: When inclusive fitness is right and when it can be wrong, J. Theor. Bio. 259, 589-600.

Weibull, J. W. [1995] Evolutionary Game Theory (The MIT Press, Cambridge, MA). 